\title{
Multiresolution Remeshing Using Weighted Centroidal Voronoi Diagram
}

\author{
Chao-Hung Lin ${ }^{1}$, Chung-Ren $\mathrm{Yan}^{2}$, Ji-Hsen Hsu ${ }^{2}$, and Tong-Yee Lee ${ }^{2}$ \\ 1 Dept. of Geomatics, National Cheng Kung University, Taiwan \\ 2 Dept. of Computer Science and Information Engineering, \\ National Cheng Kung University, Taiwan \\ http://couger.csie.ncku.edu.tw/ edirvr/
}

\begin{abstract}
We present a novel method for multiresolution remeshing of irregular mesh. First, the original mesh (two-manifold any genus) is decomposed into several patches, each patch is homeomorphic to a $2 \mathrm{D}$ triangle. The goal of this decomposition process is that the decomposed patches are size-equally. First, a mesh is manually cut into a few disk-like patches. With the help of weighted centroidal Voronoi diagram (WCVD), each patch is then automatically partitioned into more triangular patches with nearly equal size. Recursively subdividing these triangular patches, we finally get a semi-regular mesh.
\end{abstract}

Keywords: Parameterization, Remeshing, Voronoi Diagram, Resampling.

\section{Introduction}

In computer graphics and geometric modeling, 3D models are often represented by triangular meshes. The connectivity of triangular mesh produced by modeling software is usually irregular. Irregular meshes are not appropriate to handle in such tasks as model texturing and computation of finite element. Therefore, the remeshing process, i.e., modifying the sampling and connectivity of a geometry to generate a new mesh, is a fundamental step for efficient geometric processing.

The majority of previous works propose semi-regular remeshing techniques [1, 2, 3] are based on an initial step of constructing a parameterization of the original model over a base domain, i.e., so-called base mesh, consisting of a small number of triangles. A triangle in the base mesh maps to a triangular patch in the original mesh. This base mesh is then recursively subdivided, i.e., vertex resampling, to accurately fit the original mesh over the parametric domain.

Mesh re-tiling technique is proposed by [4, 5, 6] to resample the original mesh based on a particle system approach. A new set of vertices are first distributed over the surface of a given mesh and are then triangulated these vertices to create a surface that accurately fits the original mesh. A global relaxation procedure balances the distribution of the vertices by shifting them on the surface. In 6], to exactly find the sharp features, the authors extend the relaxation procedure by attracting the vertices to feature edges. 
The concept of interactive user control in geometry remeshing has been recently proposed in [7]. In [7], the original mesh is substituted by a series of $2 \mathrm{D}$ maps in parameter space. The user can combine these maps to create a control map which is used to control the vertex sampling over the surface.

The weighted centroidal Voronoi diagram (WCVD) has been applied to many applications such as non-photorealistic rendering (stippling) [13], and surface remeshing [14]. In [14, an adaptive surface remeshing technique is presented. The vertex resampling is achieved by a given density function. Utilizing the WCVD, the mesh can be adaptive tessellated. This method focuses on generating a precise isotropic surface remeshing, but it is difficult to generate a multiresolution remeshing using their remeshing scheme. Multi-resolution remeshing technique is important and can be applied to many applications such as morphing 8, 9 and LOD. In this paper, a multiresolution remeshing technique for arbitrary geometry is proposed. Unlike the previous works that create a base mesh using simplification algorithm [1,2,3, we decompose the original mesh into several triangular patches utilizing WCVD in the parametric domain. These decomposed triangular patches are nearly equal-size and similar to equilateral triangle. Once the decomposition is accomplished, these triangular patches are re-embedded onto the parametric domain to obtain a low distortion parameterization. This decomposition process can also be treated as a vertex resampling step. The next step is to recursively subdivide the triangular patches to produce a new semiregular mesh as the result.

\section{Methodology}

The basic idea of the proposed method is to decompose the original mesh $M$ into several triangular patches using the WCVD. These triangular patches are then subdivided to produce a new mesh with new connectivity that accurately fit the mesh $M$. The proposed remeshing algorithm consists of the following steps.

1. First pass decomposition: Manually partition the mesh $M$ into a few disk-like patches $\left\{P^{1}, P^{2}, \ldots, P^{m}\right\}$.

2. Parameterization: Each individual surface patch is then embedded into an isomorphic planar quadrilateral. The boundary of the parameterization is fixed to be a square so that a geometric map can be efficiently stored and processed as a regular floating point image.

3. Construct WCVD: A geometric map is computed over the parameterization and stored in 2D image as the weight of WCVD. For each quadrilateral, given $n$ sites, we construct a WCVD. The construction of WCVD will be described in more detail in Section 2.1

4. Second pass decomposition: After triangulating the WCVD, the disk-like patch $P^{i}$ is further decomposed into several nearly equal-size triangular patches $\left\{P_{1}^{i}, P_{2}^{i}, \ldots, P_{m}^{i}\right\}$. This step will be also described in more detail in Section 2.1.

5. Reparameterization: For each individual triangular patch, we re-embed it into an isomorphic planar triangle for a lower distortion parameterization. 
6. Resampling: Perform recursive 4-to-1 subdivisions on all triangular patches $P_{j}^{i}$ over the parametric domain. Finally, we can obtain a new semi-regular mesh.

\subsection{Mesh Decomposition Using Weighted Centroidal Voronoi Diagram}

Once the mesh $M$ has been decomposed into a few disk-like patches, i.e., the first pass decomposition, and embedded into planar quadrilateral, we utilize the WCVD to further decompose each disk-like patch into several triangle-like patches.

Voronoi Diagram. Given a set of points, called Voronoi sites, a Voronoi diagram partition space into several regions. For each region, any position in this region is closer to the Voronoi site of this region than to another one. In this paper, we unitize the space partitioning ability of Voronoi diagram to decompose surface patch. We adopt a fast 3D graphics hardware-based algorithm [10] to compute a Voronoi diagram. This algorithm draws a set of cones with their apexes at each site. Each cone is given a unique color as the site's identity. For each pixel, the z-buffer determines which cone is closer to the viewer and assigns the color of this cone, i.e., the site's identity, to it. Finally, we can obtain each region by the colors. This technique allows us to compute a discrete Voronoi diagrams extremely fast.

Centroidal Voronoi Diagram. To uniformly decompose the surface patch, a centroidal Voronoi diagram (CVD) is more suitable. The CVD has the property that each site lies exactly on the centroid of its Voronoi region. The centroid of a region is defined as equation 1 .

$$
C=\frac{\sum_{x_{i} \in R} x_{i}}{n}
$$

Where $R$ is the Voronoi region, is the pixel in the region $R$ and $n$ is the number of pixels in the region. Specifically, a centroidal distridution of the sites is useful because the sites are evenly distributed on the defined plane and the Voronoi region are nearly size-equally (the CVD is an optimization in some sense). These properties are appropriate to handle uniform resampling or uniform decomposition. In this paper, we adopt a relaxation algorithm 11 to produce a CVD. This algorithm can simply be stated as follows: compute Voronoi Diagram, find centroid of each region and move site to the centroid. This process is terminated when the positions of these sites are convergent. Figure 1 shows an example of CVD with 100 sites randomly placing in an equilateral quadrilateral. Figure 1(a) is the Voronoi diagram of the given sites. After 23 iterations, we obtain the CVD as shown in Figure 1(e).

Weighted Centroidal Voronoi. Diagram The CVD is created in the parametric domain which causes the regions in the planar quadrilateral to be distorted. 


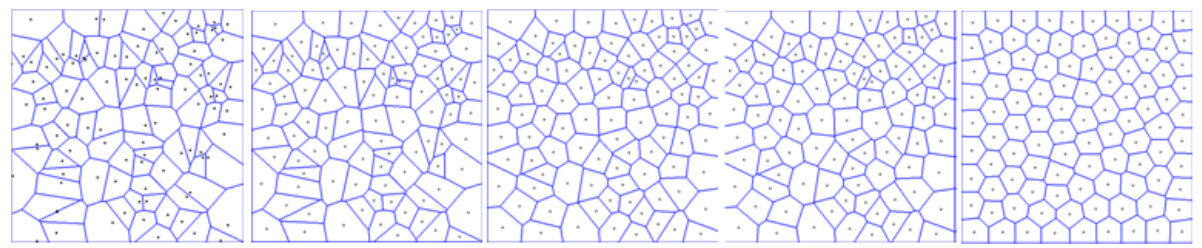

(a)

(b)

(c)

(d)

(e)

Fig. 1. Centroidal Voronoi Diagram. (a) voronoi diagram ;(b) 5 iterations ; (c) 10 iterations ; (d) 15 iterations ; (e) 23 iterations.

In this paper, we apply a weight function to the centroidal Voronoi diagram to solve this problem. The Eq. 1, i.e., calculate the Voronoi centroid, is rewritten as follows:

$$
C=\frac{\sum_{x_{i} \in R} x_{i} w_{i}}{\sum w_{i}}
$$

Where $w_{i}$ is the weight of $x_{i}$. We identify a relevant geometrical value: area distortion map $M_{A}$ to serve as the weights of CVD. The map $M_{A}$ is easily computed using the ratio $A_{3 D} / A_{2 D}$ of each triangle in the parameterization. Where $A_{2 D}$ and $A_{3 D}$ are the triangle area in the parameterization and its corresponding area on the $3 \mathrm{D}$ surface, respectively. After normalizing the scale $A_{3 D} / A_{2 D}$, the geometric map $M_{A}$ can be treated as a grayscale image range from 0 to 255 . For a better visual effect, we set the pixel with largest area distortion to be 0 and the pixel with the lowest area distortion to be 255. Following the relaxation algorithm by S. Lloyd [11, the process of constructing WCVD can be described as the pseudo-code below. In Figure 2 we show an example of the surface patch decomposition using WCVD. During the relaxation process, the darker regions of the geometry map have lager attraction for sites. With the help of WCVD, the surface patch is uniformly decomposed (as shown in Figure 2(e)). One disad-

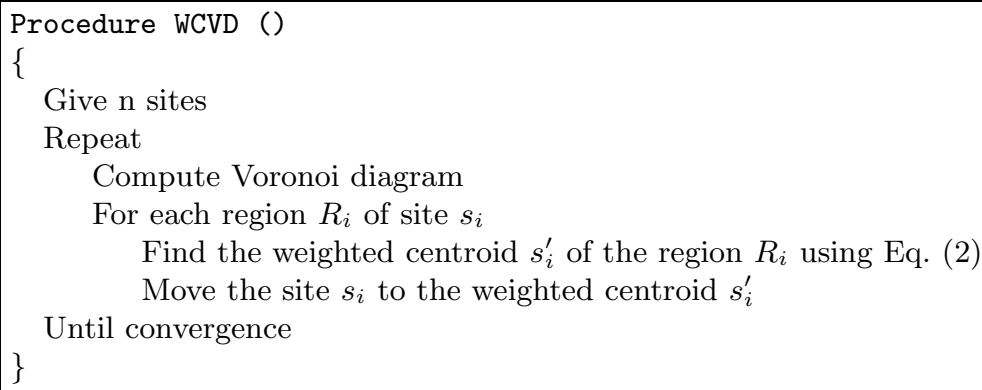

vantage of the proposed method is the use of discrete calculation of the Voronoi diagram that is affected by the resolution of the diagram and the distortion of the parameterization. A related problem is that if the resolution is too low, the sites will not distribute over the areas with an extremely high distortion. A solution described in [10] is to split the diagram into tiles and compute each tile at 


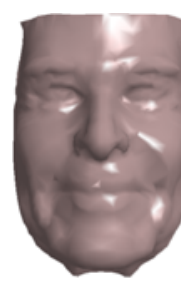

(a)

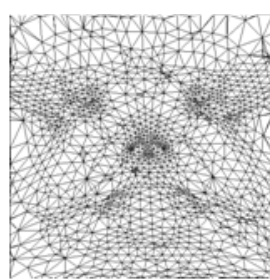

(b)

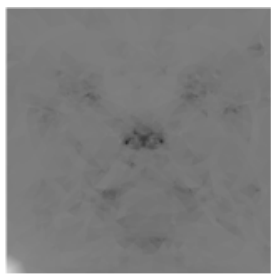

(c)

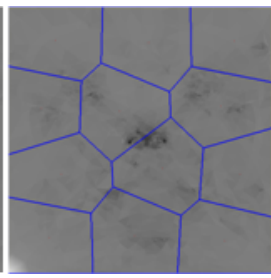

(d)

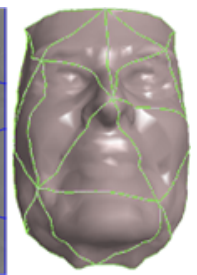

(e)

Fig. 2. Surface patch decomposition using WCVD. (a) Surface patch; (b) The parameterization of (a); (c) The area distortion map of (b); (d) The WCVD with 12 sites; (e) The surface decomposition result.

the higher resolution (increase arbitrary) and then stitch the full diagram back together. Another solution is to repartition the surface patch and then re-embed these sub-patches to parametric domain until the distortion is less than a defined threshold. In this paper, we compute a WCVD in a high resolution plane. If the problem described above still occurs, we will repartition the surface patch.

\subsection{Mesh Remeshing}

Once the decomposition process is completed, we re-embed each triangle patch to parametric domain to obtain a lower distortion parameterization. Then, we produce a new mesh with subdivision connectivity from the parameterization. The simple strategy we adopted is to perform a 4-to-1 split that the position of the split point is at the middle of an edge.

\section{Experimental Results}

In Figure 3 and 4, we show the preliminary remeshing results using the proposed method. Follow the evaluate function in $[12$. Accuracy is measured as Peak Signal to Noise Ratio $P S N R=20 * \log ($ peak $/ d)$, where peak is the diagonal length of the bounding box and $d$ is the symmetric RMS Hausdorff error (geometric distance) between the original mesh and the remesh. The Table 1 shows the Statistics of PSNR for the remeshing in the Figure 3 and 4. Roughly speaking, the PSNR about $70 \mathrm{~dB}$ is considered to be a nice approximation.

\section{Conclusion and Future Work}

We have introduced a novel approach for multiresolution uniform remeshing. Utilize the weighted centroidal Voronoi diagram to uniformly distribute the vertices in the surface and then decompose the surface to several triangular patches with nearly equal size. In the further work, we will address the processing of consistent remeshing of several different poses models. This would be very useful in the morphing and animation issues. 

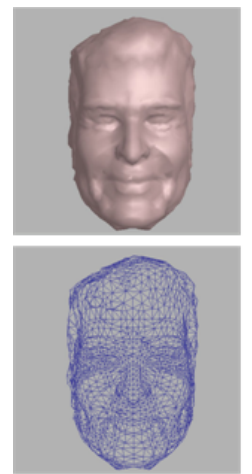

(a)
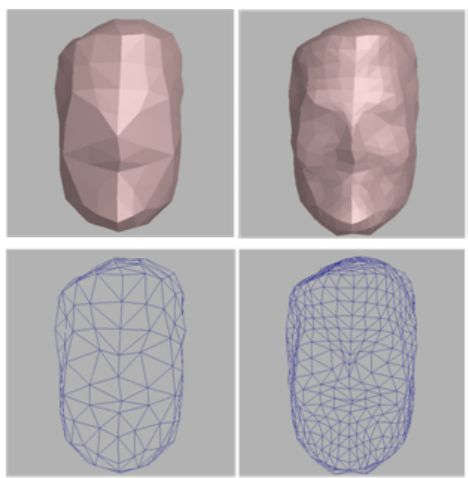

(b)

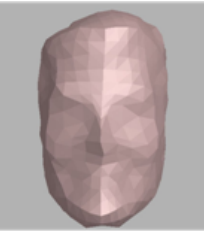

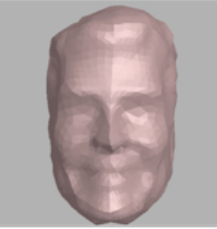
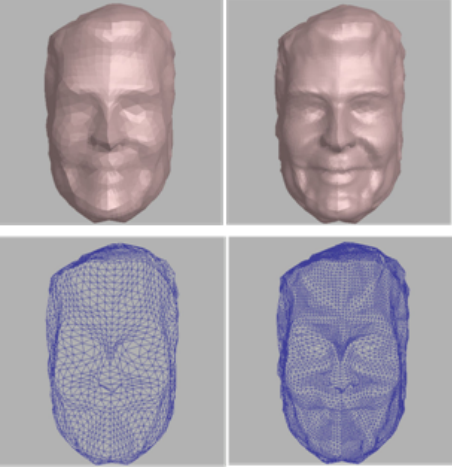

(d)

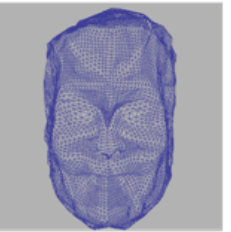

(e)

Fig. 3. Mesh remeshing of a man's head model. (a) The original mesh; (b) The decomposition result (the coarsest level of subdivision: 353 triangles); (c),(d),(e) Remeshing with 1408 triangles (level 1), 5632 triangles (level 2) and 22528 triangles (level3), respectively.
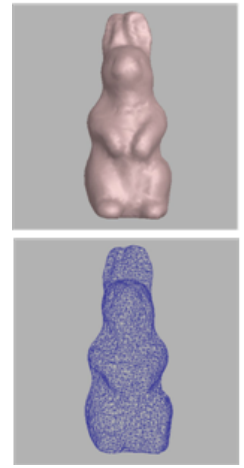

(a)

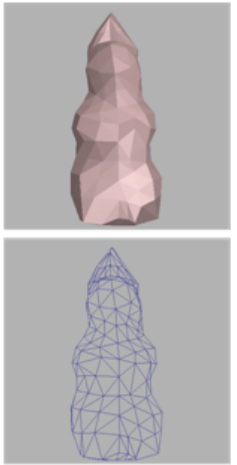

(b)

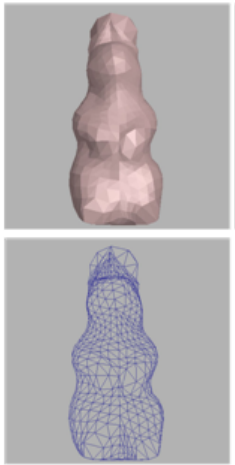

(c)

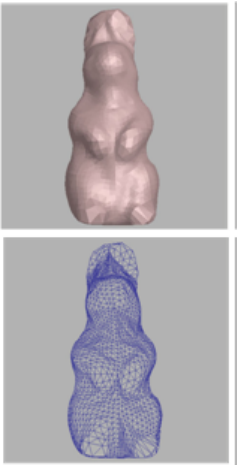

(d)

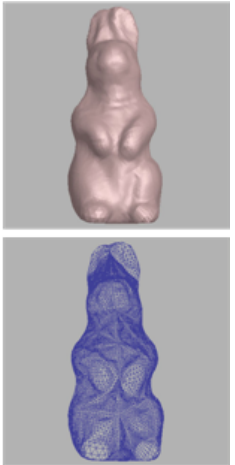

(e)

Fig. 4. Mesh remeshing of a rabbit model. (a) The original mesh; (b) The decomposition result (the coarsest level of subdivision: 472 triangles); (c),(d),(e) Remeshing with 1888 triangles (level 1), 7552 triangles (level 2) and 30208 triangles (level3), respectively.

Table 1. Statistics for PSNR

\begin{tabular}{|c|c|c|c|c|c|c|}
\hline Model & Level & Lv 0 & Lv 1 & Lv 2 & Lv 3 & Lv 4 \\
\hline Man's Head & PSNR(dB) & 44.42 & 52.81 & 60.44 & 68.40 & 85.71 \\
\cline { 2 - 7 } & no. of face & 352 & 1,408 & 5,632 & 22,528 & 90,112 \\
\hline Rabbit & PSNR(dB) & 44.40 & 50.5 & 58.71 & 61.27 & 73.96 \\
\cline { 2 - 7 } & no. of face & 472 & 1,888 & 7,552 & 30,208 & 120,832 \\
\hline
\end{tabular}


Acknowledgements. This project is supported by National Science Council, Taiwan, ROC, under contract No. 94-2213-E-006-063 and 94-2213-E-006-005.

\section{References}

1. A. W.F. Lee, D. Dobkin, W. Sweldens and P. Schroder, "MAPS: Multiresolution Adaptive Parameterization of Surfaces." ACM SIGGRAPH '98, pp. 95-104, 1998.

2. I. Guskov, W. Sweldens and P. Schroder, "Multiresolution Signal Processing for Meshes", ACM SIGGRAPH '99, pp. 325-334, 1999.

3. A. Khodakovsky, N. Litke and P. Schroder, "Globally Smooth Parameterizations with Low Distortion", ACM SIGGRAPH'03, pp. 350-357, 2003.

4. L. Kobbelt, T. Bareuther, and H.-P. Seidel "Multiresolution shape deformations for meshes with dynamic vertex connectivity", Computer Graphics Forum volume 19(3), pp. 249-260, 2000.

5. G. Turk, "Re-tiling polygonal surfaces", ACM SIGGRAPH'92, vol. 26, pp. 55-64, 1992.

6. J. Vorsatz, C. Rossl, Leif P. Kobbelt and Hans-Peter Seidel. "Feature Sensitive Remeshing", Proceedings of EUROGRAPHICS'01, pp. 393-401, 2001.

7. P. Alliez, M. Meyer and M. Desbrun, "Interactive Geometry Remeshing", ACM SIGGRAPH '02, pp. 347-354, 2002.

8. C.-H. Lin and T.-Y. Lee, "Metamorphosis of 3D Polyhedral Models Using Progressive Connectivity Transformations", IEEE Transactions on Visualization and Computer Graphics, Vol. 11, No.1, pp. 2-12, Jan./Feb., 2005.

9. T.-Y. Lee and P.-H. Hung, "Fast and Intuitive Metamorphosis of 3D Polyhedral Models Using SMCC Mesh Merging Scheme", IEEE Transactions of Visualization and Computer Graphics, Vol. 9, No.1,pp. 85-98, January-March 2003.

10. K. E. Hoff, T. Culver, J. Keyser, M. Lin and D. Manocha, "Fast Computation of Generalized Voronoi Diagrams Using Graphics Hadware", ACM SIGGRAPH '99, pp. 277-286, 1999.

11. S. Lloyd, "Least Square Quantization in PCM", IEEE Transactions on Information Theory, volume 28, pp.129-137, 1982.

12. P. Sender, J. Snyder, S. Gortler and H. Hoppe, "Texture Mapping Progressive Meshes", ACM SIGGRAPH '01, pp. 409-416, 2001.

13. A. Secord, "Weighted Voronoi Stippling", In Proceedings of NPAR, 2002.

14. V. Surazhsky, P. Alliez and C. Gotsman, "Isotropic Remeshing of Surfaces: A Local Parameterization Approach", Proceedings, 12th International Meshing Roundtable, Sandia National Laboratories, pp.215-224, Sept, 2003. 